\title{
CBS APPLIANCE: THE APPLIANCE OF CHOICE FOR DISTALIZATION OF MAXILLARY MOLARS
}

\author{
Dr. Situ Lal Shrestha, ${ }^{1}$ Dr. A. N. Chumakov, ${ }^{2}$ Dr. D.V. Bobrov, ${ }^{3}$ Dr. Alok Kumar Jaiswal ${ }^{4}$ \\ 1. Associate Prof, National Academy of Medical Sciences, Bir Hospital, Kathmandu, Nepal \\ 2. Professor, Tver State Medical Academy, Tver, Russia \\ 3. Associate Prof., Tver State Medical Academy, Tver, Russia \\ 4. Assistant Prof., MB Kedia Dental College \& Hospital, Birgunj, Nepal
}

Correspondence : c2shr@hotmail.com

\section{INTRODUCTION:}

Caries is the most common dental ailment; complications of which can lead to loss of teeth or need of premature extraction of the teeth, which in turn can lead to different morphological and functional pathologies. If the need of space maintenance is ignored, the defect can cause the neighboring teeth to shift towards the gap. It causes dental arch to shorten on the side of the gap, which in turn can cause different pathologies in eruption of permanent teeth. It can block the normal articulation of the mandible, which can lead to problems of the TMJ.

The most common complication arising from the premature extraction of the deciduous molars is the mesial shift of the permanent first molar which leads to Class II molar relation. Most commonly the canines being one of the last ones to erupt have no space in the dental arch and thus erupt out of the dental arch.

Overthe pastyears non-extraction treatment have become more popular in correction of Class II malocclusions which usually requires distalization of maxillary molars in order to achieve Class I molar and canine relationship. Various types of devices were developed for molar distalization. For years headgear was used routinely for distal movement of maxillary molars. However, headgear totally relied on patient cooperation, which could reduce treatment success and increase treatment duration. On the other hand, headgear was rejected by many patients because of aesthetic and social concern. Because of the discomfort of the patient on the use of head gear and not being possible to use continuously for all 24 hours, the treatment is prolonged, which in turn diminishes the prudence of using it to distalize maxillary molars.

The difficulties of headgear wear and dependence on patient cooperation stimulated many investigators to develop new intra-oral devices and techniques for distal movement of molars. Superelastic Ni-Ti coil springs, Pendulum appliance, Wilson's appliance, distal jet, Jasper Jumper, are all used for distal movement of the maxillary molars. In conclusion, all newly introduced intraoral distalization appliance which were developed in the last decade of the twentieth century eliminated the patient cooperation; such that, distal tipping of molar and anchorage loss are the main concerns of investigators and the orthodontists.

\section{BRIEF SUMMARY OF THE INVENTION:}

For maxillary molar distalization, CBS appliance (indicating the initials of the surnames of three authors - Dr. A. N. Chumakov, Dr. D. V. Bobrov and Dr. S. L. Shrestha), original Russian name being "ORIGINALNII APPARAT DLYA DISTALIZACIA VERKHNIKH MOLYAROV" was developed, which has been registered by the patenting authority of the Russian Federation (patent No. 45260). 


\section{DETAILED DESCRIPTION OF THE INVENTION:}

The appliance consists of a palatal support made of light-cure acrylic, strengthened with orthodontic wire (D- $0.8 \mathrm{~mm}$ ), which forms a skeleton inside the palatal support. The wire is soldered on the palatal surface of the orthodontic bands on the first maxillary premolars. On the buccal side of the band on the premolar, a cylindrical rod ( $D-0.8 \mathrm{~mm}$ ) with omega loop (medial end is fixed, distal is not) is soldered and directed towards the molar that needs to be distalized in such a way that the cylindrical rod goes into the molar tube of the molar. In between the distal end of the omega loop (which is in the form of a ring ( $D-1.0 \mathrm{~mm}$ ) encircling the cylindrical rod) and the molar tube, open NiTi coil spring (D - $1.2 \mathrm{~mm}$, $\mathrm{L}-5 \mathrm{~mm}$ ) is worn on to the cylindrical rod.

Fig. 1 : Illustration showing CBS appliance
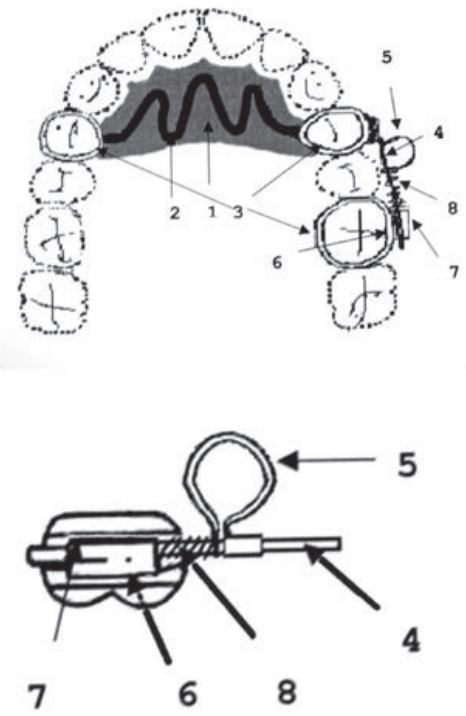

1. Palatal support (Light cure acrylic)

2. Ortho-wire (D-0.8mm)

3. Ortho-bands

4. Cylindrical rod (D-0.8mm)

5. Omega loop

6. Molar band

7. Molar tube (D-1.0mm)

8. NiTi coil spring (D-1.2mm, L-5mm)

\section{WORKING PRINCIPLE:}

A) Frontal part of the appliance consisting of palatal support (1), orthodontic bands (3) and cylindrical rod (4) with omega loop (5) is fixed with Glass lonomer Cement (GIC) on the first premolars.

B) Open NiTi coil spring (8) spirally surrounds the cylinder rod (4) till the distal end of the omega loop (5).
C) With the help of GIC, orthodontic band (6) with molar tube (7) is fixed in such a way that the cylindrical rod goes inside the molar tube.

After the band is fixed, we activate the appliance by activating the omega loop, thus activating the NiTi coilspring. Next activation is done when the NiTi coil-spring returns to the initial length $(\mathrm{L}-5.0 \mathrm{~mm})$, which takes about 2 weeks.

By activating the omega loop and thus the NiTi open coilspring, we can distalize the maxillary molar. Bands on the first premolars and the palatal support are anchorage which acts like a counter balance to the force which coil spring applies on the molar, thus preventing the anterior teeth from moving in opposite direction.

Advantage of this appliance is that it is fixed, economical easy to fabricate, and can be used in patients with mixed dentition; thus better than other appliances, which in turn gives a better results in lesser time. It can be used unilateral as well as bilateral. Average speed of the distalization of the first maxillary molars is $1.5 \mathrm{~mm}$ per month.

\section{CLINICAL CASES:}

\section{Case No. 1}

Non-extraction treatment of a patient with Class II malocclusion by bilateral distalization of the maxillary molars.

\section{History and Diagnosis}

The patient was 13 years old male (Fig 2). The medical history was negative. Dentally, he had Class II, Div 2 malocclusion with deep bite and crowding in both arches. The Steiner analysis described retrognathic mandible with an ANB of $5^{\circ}$ and a mandibular plane of $29^{\circ}$. The Down's analysis described convex profile with angle of convexity of $9^{\circ}$.
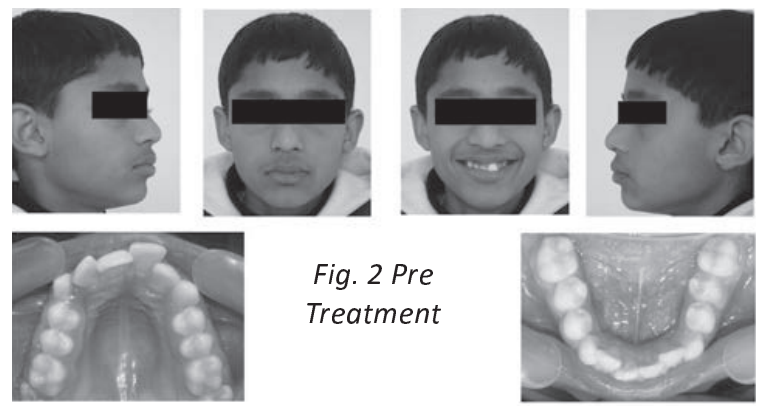

Fig. 2 Pre

Treatment
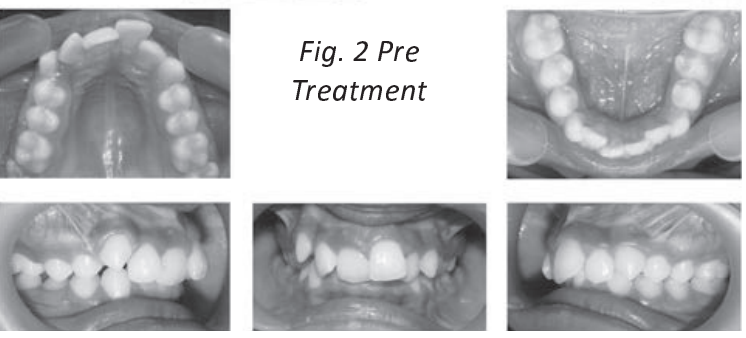

Treatment

Bilateral CBS appliance was fabricated and inserted for Distalization of the maxillary molars (Fig 3). Activation of the appliance was done every two weeks. The molars were moved distally and a Class I molar relationship was 
achieved after six months (Fig. 4). After the Distalization of the maxillary molars, Nance palatal button was fabricated and inserted for the prevention of mesial movement of the distalized molars and for providing extra anchorage for distal movement of the premolars, canines and the incisors. The patient underwent fixed orthodontic treatment using Roth prescribed .018 brackets (Fig 5). Post treatment results are shown in Figure 6.
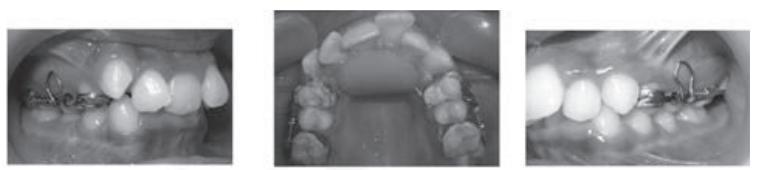

Fig. 3 Bilateral CBS appliance
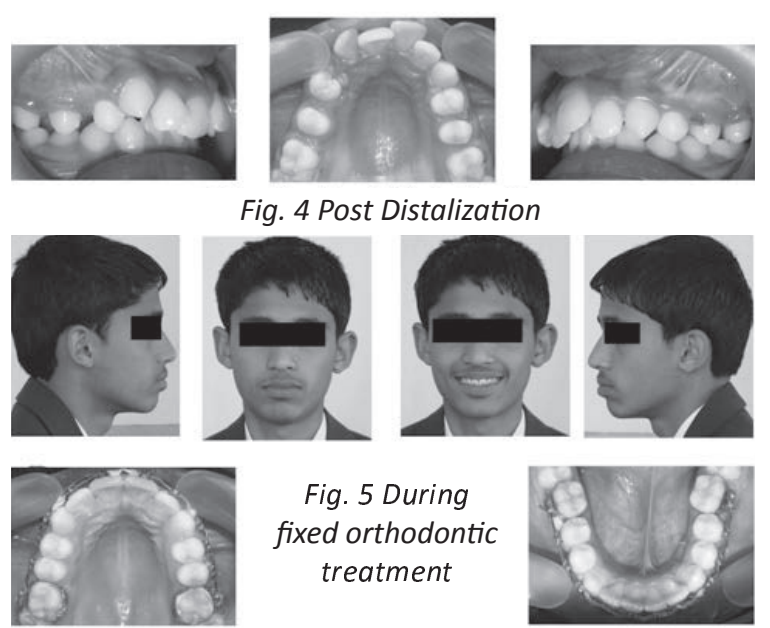

Fig. 5 During fixed orthodontic treatment
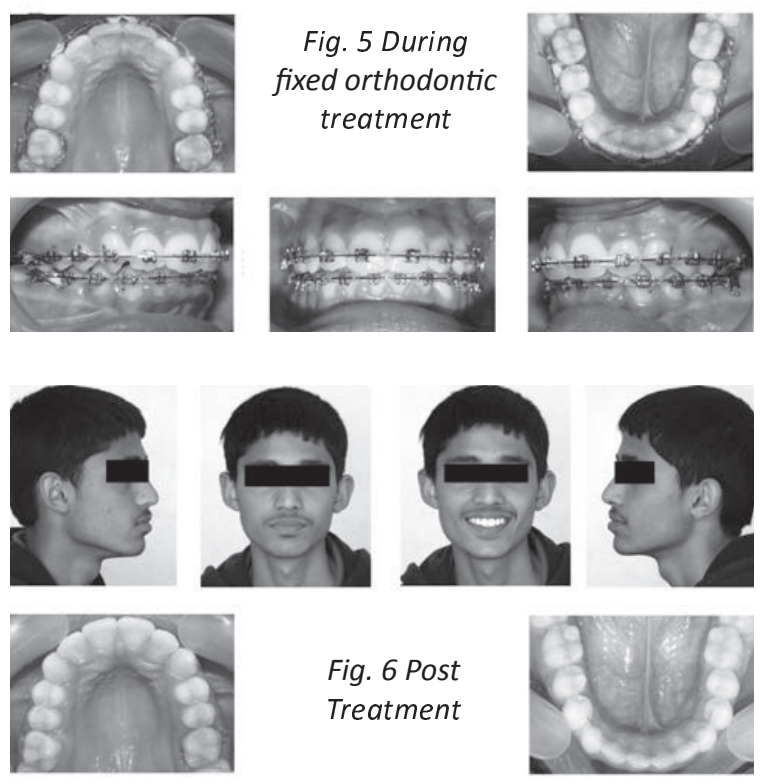

Fig. 6 Post Treatment
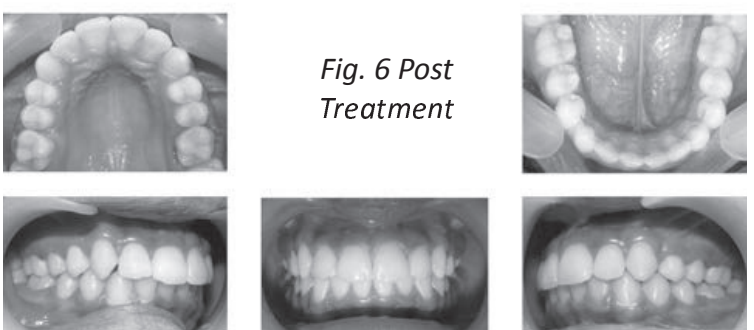

Case No. 2

Non-extraction treatment of Class II, Div 1, subdivision patient by unilateral distalization of maxillary molar.

\section{History and Diagnosis}

The patient was 21 years old female (Fig 7). The medical history was negative. Dentally, she had Class II, Div 1 subdivision malocclusion, $\mathrm{V}$ shaped upper arch with crowding in both arches. The Steiner analysis described Prognathic maxilla with ANB of $6^{\circ}$. The Down's analysis described skeletal class II, convex profile and MP angle of $23^{\circ}$.
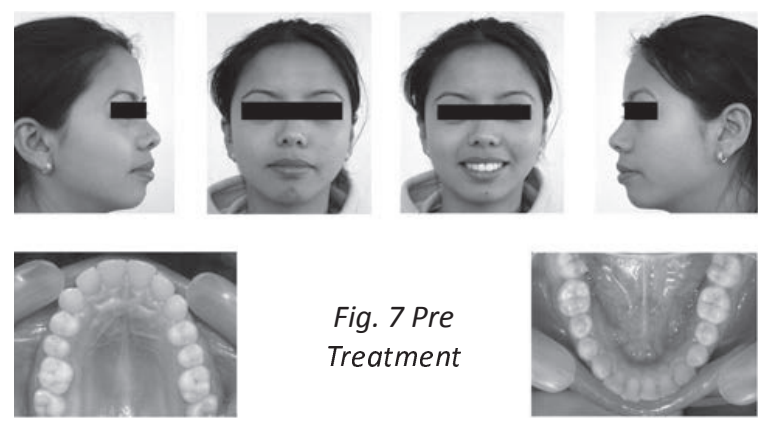

Fig. 7 Pre Treatment
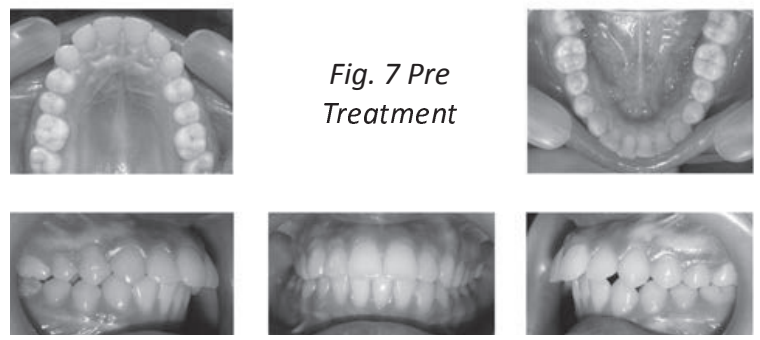

Treatment

Unilateral CBS appliance was fabricated and inserted for distalization of the right maxillary molar. Activation of the appliance was done every two weeks. The molar was moved distally and Class I molar relationship was achieved after six and a half month. After the distalization of the maxillary molars, Nance palatal button was fabricated and inserted for the prevention of mesial movement of the distalized molars and for providing extra anchorage for distal movement of the premolars, canines and the incisors (Fig. 8). Currently the patient is undergoing fixed orthodontic treatment using Roth prescribed 0.018 brackets.
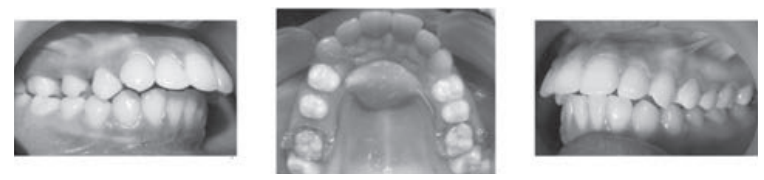

Fig. 8 Post CBS appliance

\section{Erratum:}

The case report of vol. 1 No. 1, November 2011, page 68-70 should be corrected as follows: Fig 1: Presence of mesiodens between 11 and 21, Fig 2: Radiograph of mesiodens, Fig 4: Orthodontic brackets bonded on 11 and 21 , Fig 5: Orthodontic elastic in figure of ' 8 ', Fig 6: End of space closer. 